\title{
Gold Nanoparticle Technology to Address Variability in EM Labeling
}

Richard D. Powell ${ }^{1}$, Vishwas N. Joshi, ${ }^{1}$ Frederic R. Furuya, ${ }^{1}$ Wenqiu Liu, ${ }^{1}$ John W. Dubendorff, ${ }^{1}$ James F. Hainfeld ${ }^{1}$ and Eduardo Rosa-Molinar ${ }^{2}$

${ }^{1 .}$ Nanoprobes, Incorporated, 95 Horseblock Road, Unit 1, Yaphank, NY, USA.

2. University of Kansas, Department of Pharmacology and Toxicology and Neuroscience Graduate Program, Lawrence, Kansas, USA

Electron microscopy (EM) affords a unique window into the structure and function of cells and tissues at the macromolecular level. However, in order to realize its full potential, methods are required to identify and differentiate structures of interest at a resolution, labeling density and level of multiplexing appropriate to their size, proximity and complexity.

Conventionally, colloidal gold labeled antibodies have been the method of choice for electron microscopic labeling, but suffer from limitations. Reliable conjugation protocols exist only for antibodies and a few proteins and require additional macromolecules for stabilization: these yield large probes that penetrate slowly and may only label a small fraction of closely spaced targets. When used as secondary probes especially, the "radius of uncertainty," or distance from the binding site to the gold label, may be as large as 15-25 nm or more, prohibiting the differentiation of discrete targets in small structures such as synapses. In addition, multiplexing is traditionally achieved by the use of labels of different sizes. Because each preparation of colloidal gold contains a range of sizes, multiplexing by size is generally limited to two or three targets. Furthermore, differences in probe size, which limits antigen access and labeling density, can mean that labeling results may not reflect the relative abundance of the different targets.

New and improved methods of specimen processing such as automated high-pressure freezing and freeze substitution for cryoelectron microscopy [1], availability of high-resolution STEM instruments, tomographic methods and more powerful methods for particle averaging and image analysis have created a need for labelling and target identification methods that overcome these limitations and can fully realize the power of these new methods. At the same time, current demands for rigor and reproducibility mean that the performance properties of the probes and labels should be clearly defined, consistent, and rationally attributable to their physicochemical properties and method of preparation.

We have developed an alternative approach to gold labeling in which molecular gold cluster coordination complexes, rather than colloidal gold particles, are used as the electron microscopic labels. These are stabilized by chemically defined shells of synthetic organic ligands that provide thinner, more uniform coatings than the macromolecules used to coat colloidal gold, and afford precisely controlled, monofunctional cross-linking to specific sites on a variety of biological specimens and probes using discrete cross-linking reactions via incorporated reactive groups such as maleimides and sulfo- $N$ hydroxysuccinimide (sulfo-NHS) esters. Using this approach, conjugates have been prepared using novel small molecule probes that are not amenable to colloidal gold conjugation, including antibody Fab' and smaller fragments, peptides, oligonucleotides, lipids and small molecules [2].

Furthermore, introducing controlled reactivity into the gold labels affords a means to conjugate the label directly to specific sites in biological targets, via individual chemical functional groups, such as amino acid residues in proteins, or modified nucleotides in self-assembled supramolecular oligonucleotide 
nanostructures [3]. In addition, targeting may be achieved using molecular interactions that eliminate the need for antibody or protein targeting and thus reduce the "radius of uncertainty," such as the nickel (II) nitrilotriacetic acid (NTA) moiety to target polyhistidine (His) tagged proteins. Ni-NTA-modified gold probes allow the precise labeling of His-tagged subunits in protein complexes and other specimens at nanometer resolution, affording cryoEM labeling at a precision not possible with antibody probes [4].

The $0.8 \mathrm{~nm}$ Undecagold [ $\left.\mathrm{Au}_{11}\right]$ and $1.4 \mathrm{~nm}$ Nanogold labels are molecular species, which may be differentiated from each other and from larger nanoparticles, thus expanding size-based multiplexing for the smaller gold sizes at which labeling is nearer to quantitative, but colloidal gold size variation is greatest [5]. In future, the use of precisely defined gold labels in conjunction with similarly sized nanoparticle labels of alternate composition promises to enable an additional dimension of multiplexing, expanding the complexity of the systems to which it is applicable [6].

\section{References:}

[1] KL McDonald, Protoplasma 251 (2014), p. 429.

[2] CJ Ackerson, RD Powell and JF Hainfeld, Methods Enzymol. 481 (2010), p. 195.

[3] RD Powell and JF Hainfeld, Micron 42 (2011), p. 163.

[4] CP Walsh, A Davies, AJ Butcher et al, J. Biol. Chem. 284 (2009), p. 22310.

[5] AA Sousa, MA Aronova, YC Kim et al, J. Struct. Biol. 159 (2007), p. 507.

[6] The authors acknowledge funding from the NIH (NIMH) SBIR Grant 1R43 MH106245-01.

(a)

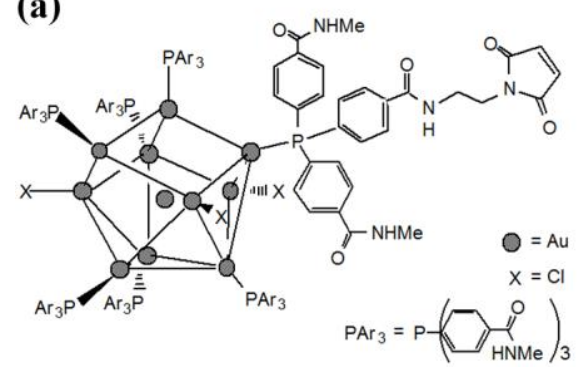

(b)

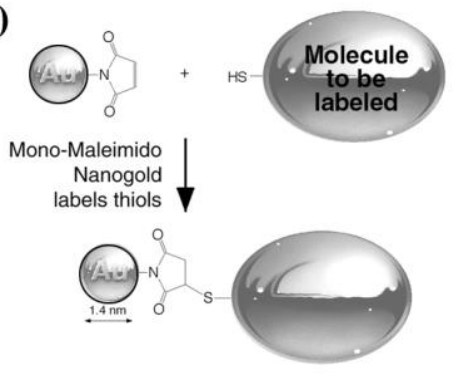

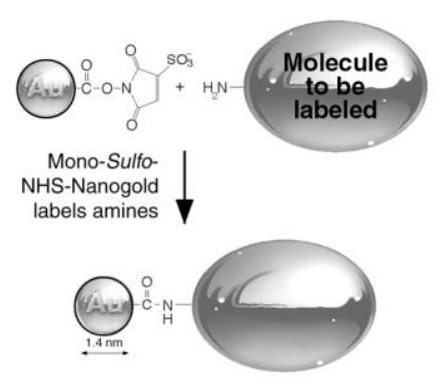

Figure 1. (a) Molecular structure of Undecagold, showing surface ligand coating and incorporation of a single reactive functionality via a coordinated ligand; (b) Labeling reactions showing selective conjugation of gold particle to specific site on conjugate biomolecule.

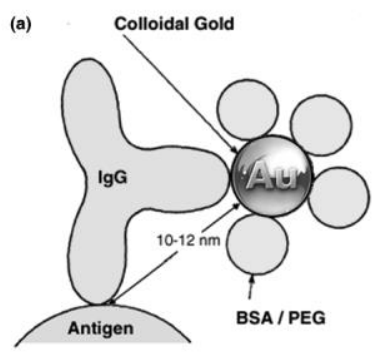

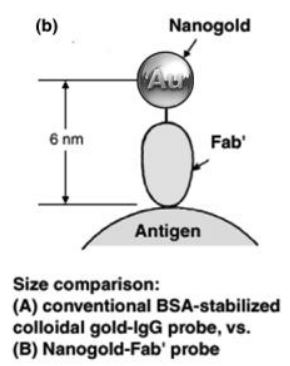

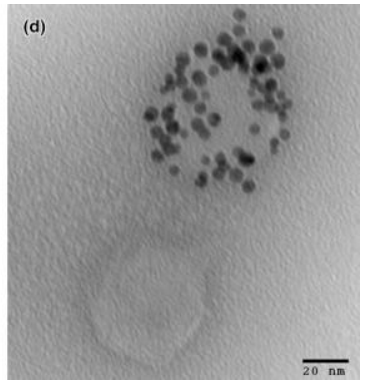

Figure 2. Comparison of labeling resolution, or "radius of uncertainty," for (a) colloidal gold-IgG conjugate, (b) Nanogold-Fab' conjugate, and (c) Ni-NTA-Nanogold. (d) $5 \mathrm{~nm}$ Ni-NTA-Nanogold labeled His-tagged T7 virus particles after incubation at room temperature for 1 hour and purification over BioGel A-5m, showing high resolution and localization of gold to the virus surface. 